\title{
RKKY MECHANISM OF SPIN INTERACTION OF LOCALIZED PROTONS IN NEUTRON STARS
}

\author{
M. Kutschera. \\ II. Niewodniczański Institute of Nuclear Physics \\ Radzikowskiego 152, 31-342 Kraków, Poland \\ AND W. WóJCIK \\ Institute of Pliysics, Technical University \\ Podchorążych 1, 30-084 Kraków, Poland
}

\begin{abstract}
We study the spin coupling of protons which are localized in dense neutron star matter. The effective spin interaction of neighbouring protons is induced by the direct spin interaction of protons with the background neutrons. The exchange integral of the effective proton-proton spin interaction is calculated. The interaction is ferromagnetic in a wide range of neutron star matter parameters.
\end{abstract}

PACS numbers: 21.65. + f, 97.60.Jd

Magnetic properties of the neutron star matter are determined by the behaviour of protons which form a dilute component of the neutron star core [1]. Protons are likely to localize at high densities $[2,3]$. This is a result of nuclear interactions which at high densities favour a single-isospin system [4, 5]. Localized protons would form a crystal structure at low temperatures [6]. Such a proton crystal has a single proton localized inside every elementary cell. As we showed in Ref. [1], neutron star matter with localized protons is unstable with respect to spin fluctuations.

It is easy to see that a single proton, which has a well-defined spin, will polarize surrounding neutron matter, as there exists direct proton-neutron spin interaction. This induced polarization of the neutron Fermi sea can in turn polarize neighbouring localized protons. This means that the RKKY (Ruderman-Kittel-KasuyaYoshida) [7] mechanism can operate and a long-range spin order can propagate in the proton crystal.

Nuclear forces are strongly spin-dependent. For our purpose here it is useful to approximate the spin potential by a contact potential with a suitably chosen coupling constant [1]. For studying a system of protons localized in potential wells due to density variation of the neutron background the relevant spin potential is 
that between the proton and the neutron. The spin potential of a proton interacting with itinerant neutrons is

$$
V=g^{\mathrm{PN}} \sum_{i} S_{i} \cdot s_{i} \delta\left(r_{1}-r_{i}\right),
$$

where the proton spin is $S_{1}$ and the itinerant neutron spin is $s_{i}$. The main contributions to this interaction come from one pion exchange, the $\rho$-exchange and the second-order tensor interaction. The above three contributions give $g^{\mathrm{PN}} \approx$ $-2.0 \mathrm{fm}^{2}$.

The localized proton wave function $\phi_{\mathrm{P}}(r)$ is assumed to be a Gaussian,

$$
\Psi_{\mathrm{P}}(\boldsymbol{r})=\left(\frac{2}{3} \pi R_{\mathrm{P}}^{2}\right)^{-3 / 4} \exp \left(-\frac{3 r^{2}}{4 R_{\mathrm{P}}^{2}}\right) \text {. }
$$

Because of the spin potential (1) every neutron from the neutron Fermi sea feels an effective field $g^{\mathrm{PN}} s_{\mathrm{P}}(r)$ due to the proton spin density

$$
s_{\mathrm{P}}(r)=n_{\mathrm{P}}(r) S_{1},
$$

where $n_{\mathrm{P}}(\boldsymbol{r})=\Psi_{\mathrm{P}}^{*} \Psi_{\mathrm{P}}$. This field induces a polarization of the neutron Fermi sea, which from the linear response theory can be calculated as

$$
s_{\mathrm{N}}(q)=-\chi(q) g^{\mathrm{PN}} n_{\mathrm{P}}(q),
$$

where $\chi(q)$ is the generalized susceptibility and $n_{\mathrm{P}}(q)$ is the Fourier transform of $n_{\mathbf{P}}(r)$. The induced spin density of the neutron Fermi sea is an inverse Fourier transform of Eq. (4),

$$
s_{\mathrm{N}}(r)=-g^{\mathrm{PN}} \frac{1}{(2 \pi)^{3}} \int \mathrm{d}^{3} q n_{P}(q) \chi(q) \exp (\mathrm{i} q \cdot r) S_{1} .
$$

The neighbouring proton, localized around a point $r_{2}$ with the spin $S_{2}$, will interact with this neutron spin density according to the spin potential (1). This interaction, which we identify with spin-spin interaction of the localized protons, is

$$
V_{\text {spin }}^{\mathrm{PP}}=-J\left(r_{2}-r_{1}\right) S_{1} \cdot S_{2}
$$

with the exchange integral

$$
J\left(r_{2}-r_{1}\right)=\left(g^{\mathrm{PN}}\right)^{2} \frac{1}{(2 \pi)^{3}} \int \mathrm{d}^{3} q \chi(q) \exp \left(-\beta q^{2}\right) \exp \left[\mathrm{i} q\left(r_{2}-r_{1}\right)\right],
$$

where $\beta=R_{\mathrm{P}}^{2} / 3$. This quantity is an inverse Fourier transform of the product of generalized susceptibility $\chi(q)$ and some Gaussian function. The spin interaction (6) is the usual RKKY indirect exchange interaction [7].

The generalized susceptibility $\chi(q)$ for a neutron gas has the form

$$
\chi(q)=\frac{3 n}{8 \epsilon_{\mathrm{F}}} F\left(q / 2 k_{\mathrm{F}}\right),
$$

where $F\left(q / 2 k_{\mathrm{F}}\right)$ is the Lindhard function,

$$
F\left(q / 2 k_{\mathrm{F}}\right)=1 / 2+\frac{k_{\mathrm{F}}}{2 q}\left(1-\frac{q^{2}}{4 k_{\mathrm{F}}^{2}}\right) \ln \left|\frac{2 k_{\mathrm{F}}+q}{2 k_{\mathrm{F}}-q}\right|,
$$

$n$ is the mean density of the neutron gas, $\epsilon_{\mathrm{F}}$ is the Fermi energy of the neutron gas and $k_{\mathrm{F}}$ is the neutron Fermi momentum. 
The exchange integral after performing the angular integration becomes

$$
J(r)=\frac{3\left(g^{\mathrm{PN}}\right)^{2} n m}{128 \pi} \frac{1}{r k_{\mathrm{F}}^{3}} \int_{-2 k_{\mathrm{F}}}^{2 k_{\mathrm{F}}} \mathrm{d} q\left(4 k_{\mathrm{F}}{ }^{2}-q^{2}\right) \exp \left(\mathrm{i} q r-\beta q^{2}\right),
$$

where $m$ is the neutron mass. For $\beta=0$ we recover the usual expression"

$$
J(r)=\frac{3\left(g^{\mathrm{PN}}\right)^{2} n m k_{\mathrm{F}}}{2 \pi}\left[\frac{\sin \left(2 k_{\mathrm{F}} r\right)-2 k_{\mathrm{F}} r \cos \left(2 k_{\mathrm{F}} r\right)}{\left(2 k_{\mathrm{F}} r\right)^{4}}\right] .
$$

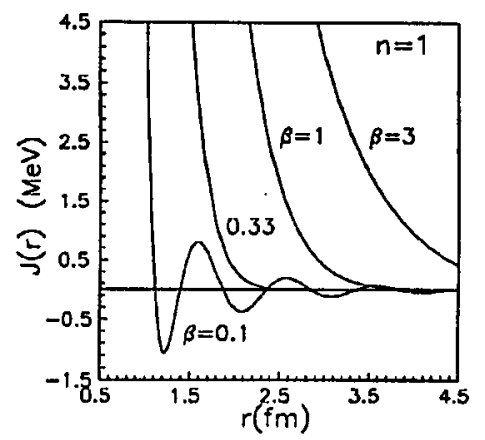

Fig. 1. The exchange integral as a function of distance between localized protons in neutron matter of density $n=1.0 \mathrm{fm}^{-3}$. The size of the probability distribution of localized proton is given by the parameter $\beta=R_{\mathrm{P}}^{2} / 3$ (in $\mathrm{fm}^{2}$ ).

We show the exchange integral $J(r)$ in Fig. 1 . One can notice that in a wide range of parameters of the neutron star matter the ferromagnetic spin ordering is favoured. In some density intervals, the oscillatory behaviour of the RKKY interaction between localized protons, displayed in Fig. 1, can lead to a variety of spin-ordered structures in the neutron stars matter. The spin structure is determined mainly by the value of $k_{F}$ and thus by the neutron density $n$. Implications for neutron stars of the proton spin interaction considered here are studied elsewhere [8].

This research is partially supportcd by the Committee for Scientific Research, grants 2 P03B 08308 and 2 P03D 00109.

\section{References}

[1] M. Kutschera, W. Wöjcik, Phys. Lelt. B 223, 11 (1989).

[2] M. Kutschera, W. Wójcik, Acta Phys. Pol. B 21, 823 (1990).

[3] M. Kutschera, W. Wojjcik, Phys. Rev. C 47, 1077 (1993).

[4] M. Kutschera, Z. Phys. A 348, 263 (1994).

[5] M. Kutschera, Phys. Lett. B 340, 1 (1994).

[6] M. Kutschera, W. Wójcik, Nucl. Phys. A 581, 706 (1995).

[7] M.A. Ruderman, C. Kittel, Phys. Rev. 96, 99 (1954); T. Kasuya, Prog. Theor. Phys. 16, 45 (1956); K. Yoshida, Phys. Rev. 106, 893 (1957).

[8] M. Kutschera, W. Wójcik, in preparation. 